\title{
BMJ Open Observational retrospective study of UK national success, risks and costs for 319,105 IVF/ICSI and 30,669 IUI treatment cycles
}

\author{
Gulam Bahadur (D) , ${ }^{1}$ Roy Homburg, ${ }^{2}$ Judith E Bosmans, ${ }^{3}$ Judith A F Huirne, ${ }^{4}$ \\ Peter Hinstridge, ${ }^{1}$ Kanna Jayaprakasan, ${ }^{5}$ Paul Racich, ${ }^{6}$ Rakib Alam, ${ }^{1}$ \\ Ioannis Karapanos, ${ }^{1}$ Afeeza Illahibuccus, ${ }^{1}$ Ansam Al-Habib, ${ }^{1}$ Eric Jauniaux (D) ${ }^{7}$
}

To cite: Bahadur G,

Homburg R, Bosmans JE, et al. Observational retrospective study of UK national success, risks and costs for 319,105 IVF/ICSI and 30,669 IUI treatment cycles. BMJ Open 2020;10:e034566. doi:10.1136/ bmjopen-2019-034566

- Prepublication history for this paper is available online. To view these files, please visit the journal online (http://dx.doi org/10.1136/bmjopen-2019034566).

Received 28 September 2019 Revised 22 January 2020 Accepted 13 February 2020
Check for updates

(C) Author(s) (or their employer(s)) 2020. Re-use permitted under CC BY-NC. No commercial re-use. See rights and permissions. Published by BMJ.

For numbered affiliations see end of article.

Correspondence to

Dr Gulam Bahadur;

bahadur.g@gmail.com

\section{ABSTRACT}

Objective To compare success rates, associated risks and cost-effectiveness between intrauterine insemination (IUI) and in vitro fertilisation (IVF).

Design Retrospective observational study.

Setting The UK from 2012 to 2016.

Participants Data from Human Fertilisation and

Embryology Authority's freedom of information request for 2012-2016 for IVF/ICSI (intracytoplasmic sperm injection) and IUI as practiced in $319105 \mathrm{IVF} / \mathrm{ICSI}$ and $30669 \mathrm{IUI}$ cycles. Direct-cost calculations for maternal and neonatal expenditure per live birth (LB) was constructed using the cost of multiple birth model, with inflation-adjusted Bank of England index-linked data. A second direct-cost analysis evaluating the incremental cost-effective ratio (ICER) was modelled using the 2016 national mean (baseline) IVF and IUI success rates.

Outcome measures LB, risks from IVF and IUI, and costs to gain $1 \mathrm{LB}$.

Results This largest comprehensive analysis integrating success, risks and costs at a national level shows IUI is safer and more cost-effective than IVF treatment. IVF LB/cycle success was significantly better than IUI at $26.96 \%$ versus $11.49 \%(p<0.001)$ but the IUI success is much closer to IVF at 2.35:1, than previously considered. IVF remains a significant source of multiple gestation pregnancy (MGP) compared with IUI (RR (Relative Risk): 1.45 (1.31 to 1.60), $\mathrm{p}<0.001$ ) as was the rate of twins (RR: $1.58, p<0.001)$.

In 2016, IVF maternal and neonatal cost was $£ 115082$ 017 compared with £2 940196 for IUI and this MGPrelated perinatal cost is absorbed by the National Health Services. At baseline tariffs and success rates IUI was $£ 42558$ cheaper than IVF to deliver 1LB with enhanced benefits with small improvements in IUI. Reliable levels of IVF-related MGP, OHSS (ovarian hyperstimulation syndrome), fetal reductions and terminations are revealed.

Conclusion IUI success rates are much closer to IVF than previously reported, more cost-effective in delivering 1 $\mathrm{LB}$, and associated with lower risk of complications for maternal and neonatal complications. It is prudent to offer IUI before IVF nationally.

\section{Strengths and limitations of this study}

- Largest aggregate UK statutory national data on in vitro fertilisation (IVF)/ICSI and intrauterine insemination (IUI) treatment cycles from Human Fertilisation and Embryology Authority gained under freedom of information overcome selection biases and ensure a high degree of applicability of the study results.

- Study uniquely describes comparative treatment outcomes, multiple birth risks, cost implications, including cost to achieve a live birth and of neonatal costs.

- Data reveal extent of other risks such as OHSS, fetal reduction, pregnancy terminations and the level of higher order multiple births.

- Retrospective and observational study.

- Limitations stem from unavailability of associated clinical data such as diagnosis or stimulation methods, distinction of IVF and ICSI cycles, and fresh or frozen IVF cycles to go alongside the baseline IUI data.

\section{INTRODUCTION}

There is limited comprehensive and direct comparison between intrauterine insemination (IUI) and in vitro fertilisation (IVF) treatments at national level encompassing success, risk and cost.

Information on different assisted reproductive techniques (ART) success, risks, failures and costs can be challenging for patients, politicians and healthcare stakeholders paying for services. IVF success claims have an overbearing presence on social media and in-patient forums to such an extent that other ART such as IUI are relegated or dismissed outright. The Human Fertilisation and Embryology Authority (HFEA) website ${ }^{1}$ appears wholly negative towards IUI suggesting poor success, uncontrolled multiple births and a high cost of failure over several cycles. 
The UK National Institute of Health and Care Excellence (NICE) ${ }^{2}$ examined the effectiveness of $25 \mathrm{mg}$ clomiphene citrate with IUI against expectant management and found no differences, but then without comparative IUI and IVF evidence recommended removing IUI altogether and replacing it with three cycles of IVF. ${ }^{23}$

A recent systematic review and meta-analysis including 10 randomised controlled trials (RCTs) comparing IUI and IVF found no difference in safety and effectiveness between treatments. ${ }^{4}$ The overall quality of the evidence provided by RCTs has been low or very low for all comparisons. ${ }^{4}$ Subsequent well-designed RCTs support IUI over IVF. ${ }^{5-8}$

There are no large national data analyses. A European meta-analysis relying on voluntary data submission identified 1 million under-reported cycles from 6 million cycles which would compromise interpretations of the data. ${ }^{9}$ Statutory national data analysis removes the selection bias of individual studies and the incompleteness of voluntary data submissions.

We aimed to compare the effectiveness of IUI with IVF as practiced in the UK over a 5 -year period where full data were available. The hypothesis tested was that IUI would be associated with better outcomes than IVF in terms of pregnancy success rates, risk of complications and costs.

\section{METHODS}

\section{Study design and participants}

This is a retrospective observational study of $319105 \mathrm{IVF} /$ ICSI and 30669 IUI cycles performed between 2012 and 2016 in the UK. DI (donor insemination) cycles were excluded. Data were obtained from the UK-regulated HFEA database. The data were collected entirely by HFEA staff under freedom of information (FOI) including live birth (LB) per cycle, multiple births, OHSS, fetal reduction, terminations and level of government funded IVF cycles for IVF and IUI. Other clinic data were available on the HFEA website. ${ }^{10}$

Direct cost for maternal and neonatal expenditure per LB was constructed using the cost of multiple birth (COMBS) model as previously described, ${ }^{11}$ with inflationadjusted pricing using Bank of England index-linked data. The final figures were derived from the number of IUI and IVF cycles performed in 2016, against the level of multiple gestation pregnancy (MGP) produced. Twin and triplet analyses allow for an individualised cost calculation for every birth component to be applied evenly and accurately for maternal and neonatal care based on the COMBS model.

A second direct cost analysis evaluating the incremental cost-effective ratio (ICER) ${ }^{12}$ was modelled on the 2016 national mean (baseline) IVF and IUI success rates. An algorithm was developed to establish the relationships between IUI and IVF at prevailing baseline live birth rate (LBR) and costs to determine cost-effectiveness from clinics with variable success rates.
The relationship between IUI and IVF clinics with high LBR was considered alongside the standard performing clinics using the HFEA database and for IUI additional in-house (North Middlesex University Hospital, NMUH) data were available. The calculations were also performed using the mean 2016 IVF tariff. ${ }^{13}$ The common tariffs per treatment cycle for IUI at $£ 800-£ 1300$ and $£ 3500-5000$ (HFEA mean $£ 4699 /$ cycle) were entered into our algorithm model, while IVF outliers for success and tariffs were considered separately.

\section{Randomisation and masking}

The very large data relate to actual and overall national practice and override RCT requirements for stratification or masking where small numbers are considered. RCTs are also associated with selected population and involve experimental design to control external factors.

Limitation of the UK FOI data set is the absence of information on ovarian stimulation regimes, number of patients treated, mean number of treatment cycles per patient, cause of subfertility, age of patient, use of fresh or frozen IVF cycles, the potential benefit and cost of transferring cryopreserved embryo in subsequent cycles, extent of eSET, previous ART, oocyte retrieval complications, birth weight, sex ratios, gestational age and ectopic and molar pregnancies. Repeated FOI request could generate small differences in data given the enormity of the data gathering exercise. Unfactored also are the costs due to OHSS, fetal reduction, termination, sperm/ embryo freezing, embryo culture, add on techniques and work absenteeism. There was no distinction between IVF and ICSI cycles.

A general diagnosis for 2012-2016 of male infertility $(37 \%)$, unexplained $(32 \%)$, ovulatory disorder $(13 \%)$, tubal disease $(12 \%)$ and endometriosis $(6 \%)$ was provided for IVF/ICSI only.

\section{Procedures}

The procedures are fully described under methods section.

The procedure of data collection was through FOI requests from the UK HFEA collecting information as part of its regulatory remit and for the purpose of issuing a licence to clinics to operate. There is therefore no scope for clinics to selectively submit or withhold their activities or outcome data.

The HFEA provided a summary of the data in tabulated form which also restricts further interrogation of data or the manner in which statistics can be performed. The HFEA also provided details to reveal the nature of pregnancy terminations.

None of the authors could influence the data set or the manner in which data could further inclusion/exclusion choices, as this was handed over under FOI by the HFEA. No UK clinic could selectively submit data to the HFEA. Submission is a statutory obligation, resulting in revocation of the clinic licence for serious breaches. 


\section{Outcomes}

The primary outcome was LB per cycle, MGP levels, OHSS, fetal reduction and terminations. The reasons for terminations were disclosed. The total cost of maternal and neonatal care through IUI and IVF for 2016 was calculated using actual 2016 figures derived from the COMBS model ${ }^{11}$ and inflation adjusted figures from the Bank of England data. ICER, to deliver $1 \mathrm{LB}$, was obtained for real IUI and IVF activities and actual tariffs paid by patients. The percentage of IVF cycles was disclosed by the HFEA, which revealed the level of funded treatments performed by NHS clinics and NHS-funded private IVF clinics (which arenot accountable under FOI laws).

\section{Statistical analysis}

The proportions with the SE were calculated for the given outcome. The $95 \%$ CI was calculated as +/-1.96 SE. Absolute and relative differences in risk were calculated, together with their SE. P values were calculated from the ratio of the measured absolute difference to the SE, assuming variation to be normally distributed. Analysis was performed for main outcome measures such as LB rates, MGP rates, market worth of interventions against maternal and neonatal costs, and cost to achieve 1 LB and permutation thereof. Within IVF and IUI activity trends were calculated using Graphpad Prism software V.8. For each exposure and outcome studied, relative risks were calculated using Medcalc software (https://www.medcalc. org/calc/relative_risk.php). For each comparison, the $\mathrm{SE}$ was calculated for the difference in absolute risk, and a two-tailed $z$-score was calculated. $5 \%(\mathrm{p}=0.05)$ was taken as the limit of significance.

\section{RESULTS}

Data from the UK HFEA database between 2012 and 2016 showed an overall 10.4-fold increased use of IVF compared with IUI. The downward trend in IUI cycles was statistically significant $\mathrm{R}^{2}$ of $0.89(\mathrm{p}<0.05)$ (table 1$)$, whereas there was a statistically significant increase in IVF cycle showing a significant slope $\mathrm{R}^{2}$ of $0.99(\mathrm{p}<0.001)$ (table 1).

There was a statistically significant improvement in LBR for IVF between 2012 and 2016 [2012: 25.47\% (25.1225.82); 2016: $27.32 \%$ (26.98-27.65)]. The absolute difference was $1.84 \%$ (1.36-2.33), RR: 1.07 (1.05-1.09), $\mathrm{p}<0.001$. The improvement from $25 \%$ to $27 \% \mathrm{LB} /$ cycle for IVF remains small.

There was no change in IUI success over the same period [2012: $11.66 \%$ (10.93-12.39); 2016: $12.10 \%$ (11.09-12.10)]. The absolute difference was $0.43 \%$ (0.81-1.67). RR: $1.04(0.93-1.15) \mathrm{p}=0.25$ (table 1). IVF had a significantly higher LB/cycle compared with IUI [IVF: 26.96\% (26.81-27.12); IUI: 11.49\% (11.13-11.85)]. The absolute difference was $15.47 \%$ (15.09-15.86). RR: 2.35 (2.27-2.42), $\mathrm{p}<0.001$. An additional birth will be achieved for every 6.5 IVF cycles compared with IUI $($ NNT $($ Number Needed to Treat $)=6.46)($ table 1$)$.

The rate of MGP as a proportion of all births was significantly higher after IVF than IUI [IVF: $13.88 \%$ (13.6514.11); IUI: $9.59 \%$ (8.62-10.56) ] with absolute difference: $4.29 \%$ (3.29-5.29). RR: 1.45 (1.31 to 1.60 ), $\mathrm{p}<0.001$. An additional multiple pregnancy will occur for every $23 \mathrm{IVF}$ pregnancies compared with IUI (NNT=23.31) (table 1).

Data from 2012 to 2016 (table 1) reveal other risks such as OHSS, fetal reduction and terminations and the level of government funded IVF cycles and additionally the level commissioned through private IVF clinics. As part of the risk factor association, reasons for terminations were additionally disclosed by the HFEA. Results from table 1 are discussed in detail.

\section{Financial calculation}

Two types of financial analyses are included: (a) the national maternal and neonatal care cost burden and (b) ICER, to deliver $1 \mathrm{LB}$ per cycle (figures 1 and 2).

\section{Size of maternal and neonatal cost}

Based on HFEA 2012-2016 figure 1 LB from IUI is made up of singleton: twin: triplet in the components 91.25: 7.90: 0.85 , respectively, whereas for IVF the singleton component is reduced in the proportion 86.21: 13.65: 0.14 . Twin and triplet figures were number of sets, rather than individual babies. For 2016, maternal and neonatal, adapted from COMBS model and index link adjusted for 2016, the cost $(£)$ per birth events factoring the singleton, twin and triplet cost was $£ 4945, £ 13618$, and $£ 48$ 300, respectively. Associated maternal and neonatal cost for one IUI and IVF baby was $£ 6000.406525$ and $£ 6186.538342$, respectively.

\section{Financial size of the 2016 UK IVF and IUI industry against the MGP risk cost burden for the NHS}

The IVF maternal and neonatal cost for 2016 was $£ 115$ 082017 against the IVF market value of £238 346 500-340 495000 for 2016. In contrast, for IUI the maternal and neonatal risk burden from IUI was £2 940196 against the IUI market value of $£ 3,240,800-5,266,300$. The maternal and neonatal risk burden due to IVF is 39 -fold higher than IUI, against a 10.4-fold greater IVF activity over IUI. The size of maternal and neonatal care for the UK from IVF or IUI could not be confirmed by the Department of Health and Social Care's (FOI response FOI-1160477).

To understand the relationships between high LBR IUI and IVF clinics, data from NMUH IUI at $£ 800$ tariff were utilised. Between 2014 and 2018: 672 cycles, 364 women, 119 pregnancies, 2 twins; PR (pregnancy rate)/cycle $17.7 \%$ /cycle; PR/woman 32.7\%/woman; LB/woman $28.7 \%$ /woman; LB/cycle $15.60 \%$; two twins, no severe to moderate OHSS reportable to the HFEA, but seven cases of mild-moderate OHSS not requiring hospitalisation; $63 \%$ of all cycles performed using consecutive ejaculation, ${ }^{14}$ hMG (human menopausal gonadotropin) stimulated with strict cancellation policy for $>=3$ follicles or 


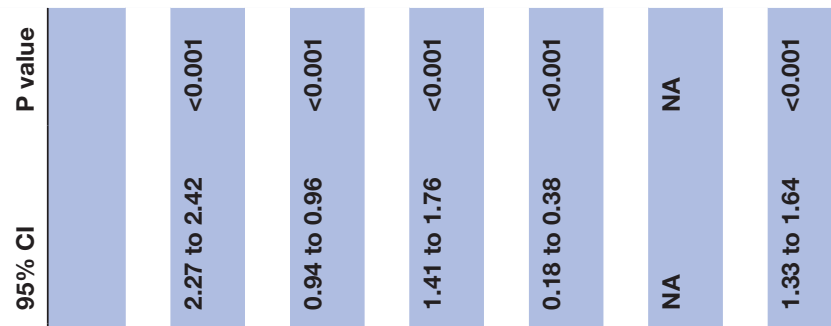

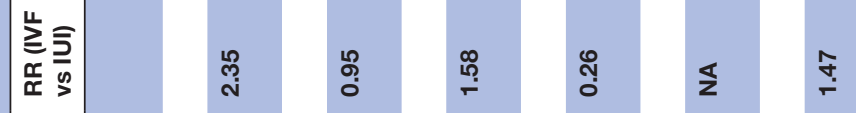

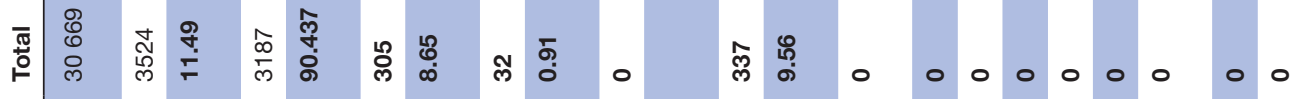

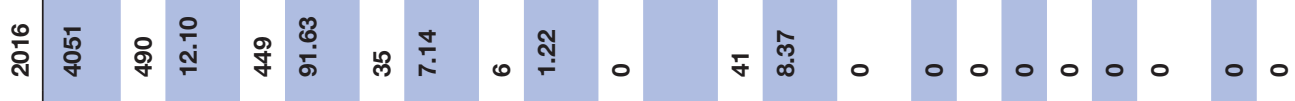

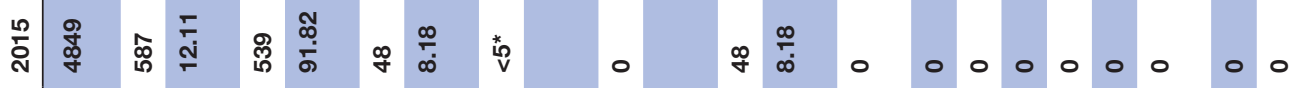

节热

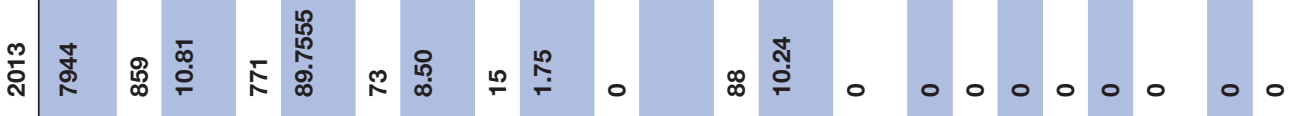

商点

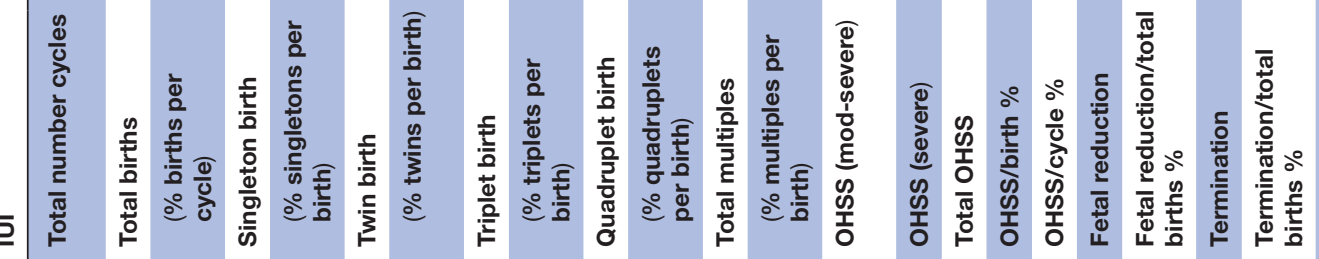

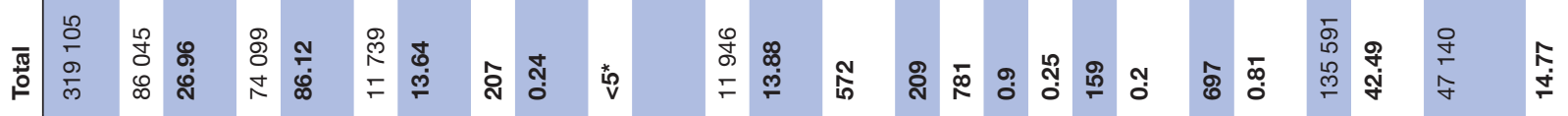

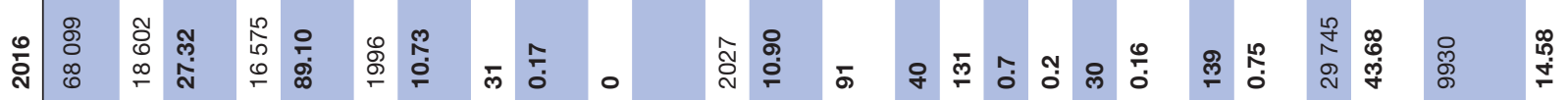

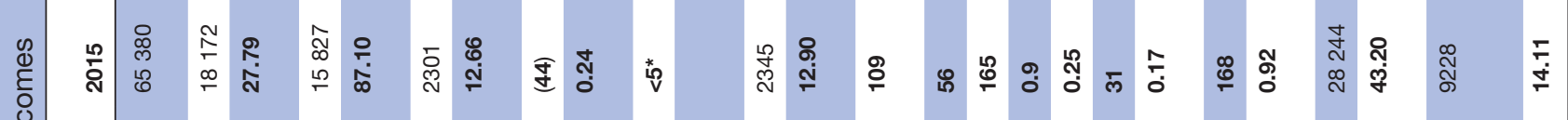

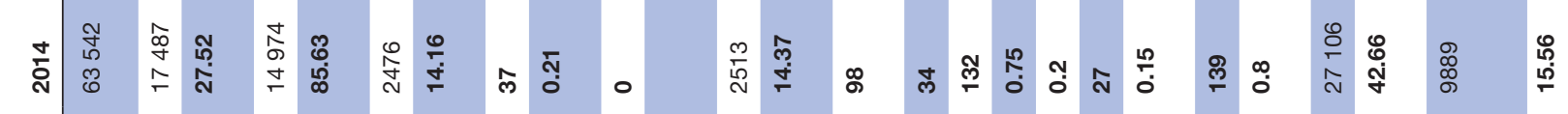

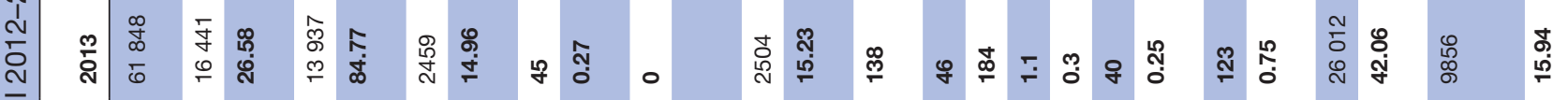

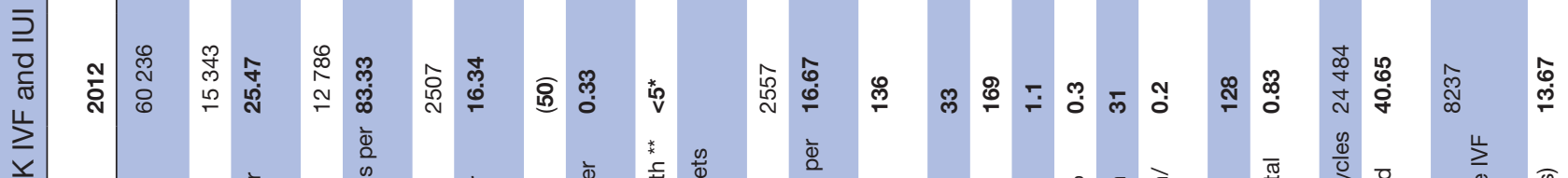

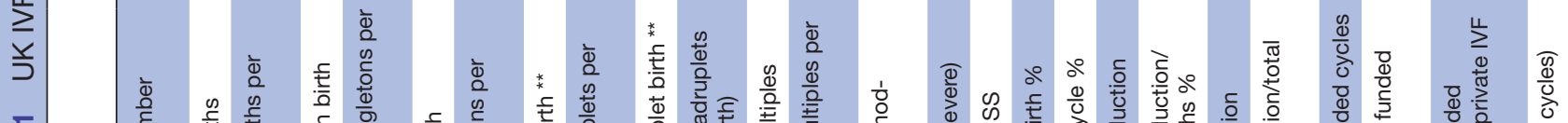

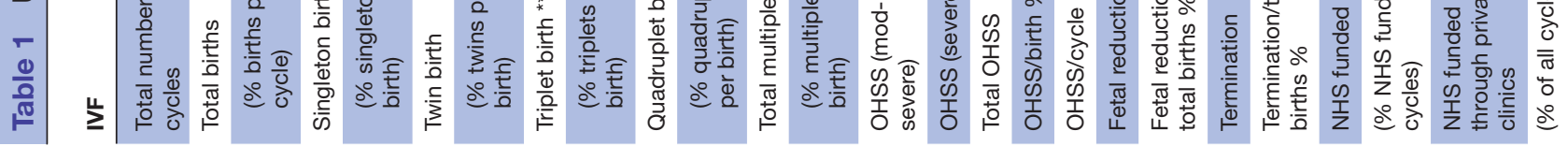




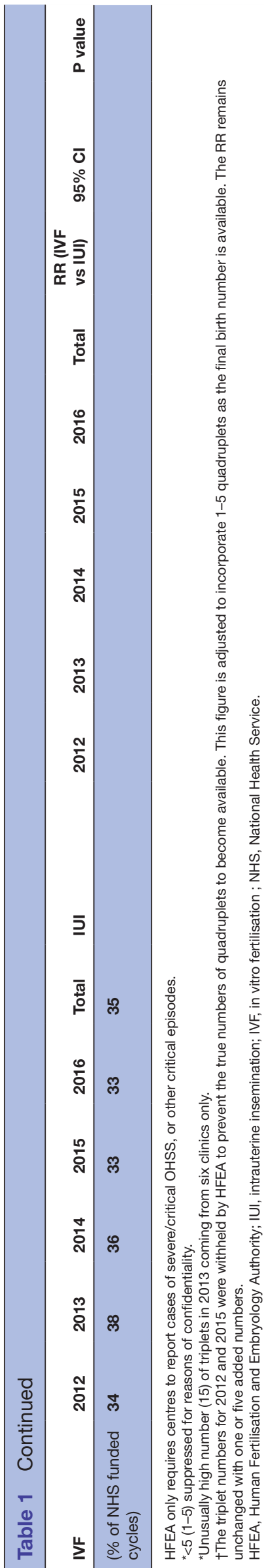

OHSS. Against this, typical higher undisclosed IVF clinic results showed $55 \% \mathrm{LB} /$ cycle and 30\% MGP rates from the HFEA database, ${ }^{10}$ and which also had typical IVF tariff of £7000-15 000/cycle. Both sets of data were placed in perspective against standard IVF clinics where the mean success rate for 2016 was $27 \% \mathrm{LB} /$ cycle and with a mean tariff of $£ 4699$.

\section{DISCUSSION}

These data add to previously published studies $^{5-8}$, ${ }^{15-17}$ showing better LBR following IVF than with IUI but this difference is much smaller than previously reported. Our study has also identified new data on the use of IVF and IUI in the UK which should have a direct impact in management strategies of couples presenting with infertility. Data summary provided by the HFEA has also allowed us to perform descriptive comparisons and trends, but limits the use of complex statistics. This is advantageous as alternative interpretations of the data become impossible, thereby removing interest biases. No sample size calculation was required to determine the power at a significance level of $0 \cdot 05$.

During the period of our study, IVF was associated with a significantly $(\mathrm{p}<0.001)$ higher risk of MGP than IUI. IVF is also associated with higher risks of OHSS and need for fetal reduction and pregnancy terminations which were not found after IUI. We found that from 2012 to 2016, there was 10.4-fold increase in the use of IVF over IUI with a significant $(\mathrm{p}<0.05)$ increase in IVF cycles and a corresponding decline in IUI cycles (table 1). This increased IVF activity cannot be justified on the basis of evidence-based data, since there are no comparative IVF versus IUI data. ${ }^{3}$ The increase in the number of IVF and decline in IUI cycles (table 1) coincide with the 2013 NICE guidelines ${ }^{3}$ recommendation against the use of IUI as the primary therapeutic line for infertile couples. This erroneous recommendation was based on poor IUI outcomes compared with expectant management with no data on IVF and yet recommended IVF practice instead of IUI. ${ }^{3}$ Our data show a small increased but significant $(p<0.05)$ LBR following IVF from $25 \%$ to $27 \%$ during that period following the NICE recommendation.

The baseline IUI: IVF success rates (table 1) to deliver 1 LB was 2.35:1, which was much narrower than the RCT reported of 3:1 for IUI: IVF. ${ }^{5-8} 15-17$ Therefore, a small improvement in IUI LBR from $12.1 \%$ to $15.6 \%$ LBR narrows this difference to 1.73: 1, IUI: IVF cycles, with a highly favourable cost benefit for IUI over IVF. When considering IVF as a denominator it is important to emphasise that 3.7 IVF cycles are actually required to achieve a $100 \%$ theoretical LB. Overall, we found 8.69 IUI cycles at $12.1 \%$ LBR or 6.4 cycle for a $15.6 \%$ LBR IUI NMUH clinic are needed to theoretically achieve $100 \%$ LB. Despite the focus on IUI against IVF, the relationship between ordinary and high success IVF clinics seems curiously avoided. Mean-IVF clinics (27\% LBR, MGP 15\%) against as high performing IVF clinic (55\% LBR, MGP 


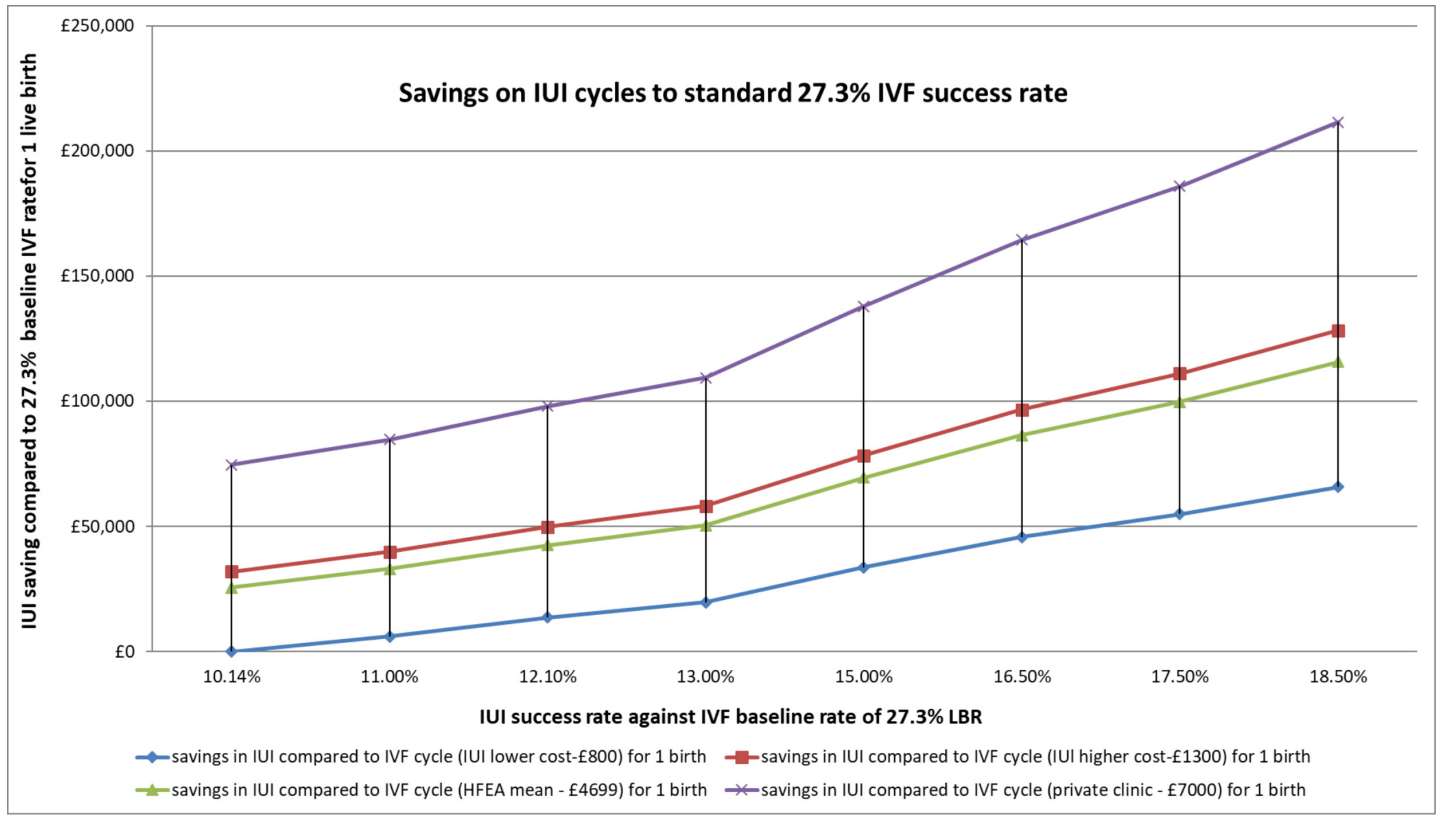

Figure 1 Economic and financial analyses of IUI versus IVF- direct cost-effective analyses to deliver 1 LB based on actual success rates and tariffs for IUI and IVF. Cost analyses with varying IUI success against mean IVF success.

$30 \%)$ have a 2.04:1 relationship but higher IVF clinics have riskier (2.2:1) outcomes for mother and baby.

Factoring tariffs alone, IUI is cheaper than IVF while also delivering lesser risks and perinatal complications for both mothers and babies. Higher IVF success clinics lose any benefits through increased tariffs and doubled MGP levels against IUI and against standard performing IVF clinics. The actual cost benefits are displayed in figures 1 and 2 which study the ICER to deliver $1 \mathrm{LB}$ at various success rates and tariffs. Against prevailing tariffs IUI always has lower overall costs than IVF in commissioning treatment to achieve $1 \mathrm{LB}$. Patients should be informed that IUI success is closer to IVF but without the added risks to mothers and babies, the cost burden to the healthcare system of which are excessive for IVF.

A recent RCT shows that after 3-4 years unexplained infertility randomised for three cycles of IUI (CC) or expectant management ${ }^{5}$ a three-fold improvement in outcome in LBR from $31 \%$ and $9 \%$ is seen. IUI LB/cycle probabilities ranged from $21.4 \%$ to $5.1 \%$ dependent on age, cycle number and previous parity, with a MGP of $5.4 \%{ }^{18}$ IUI was also effective on LBR (OR 1.95 (1.10 to 3.44$)(95 \% \mathrm{CI})$ ) when compared with intercourse or expectant management in a stimulated cycle. ${ }^{19}$ Currently in most clinics, IUI suited cases are now receiving IVF treatments or are further aided with non-evidenced based

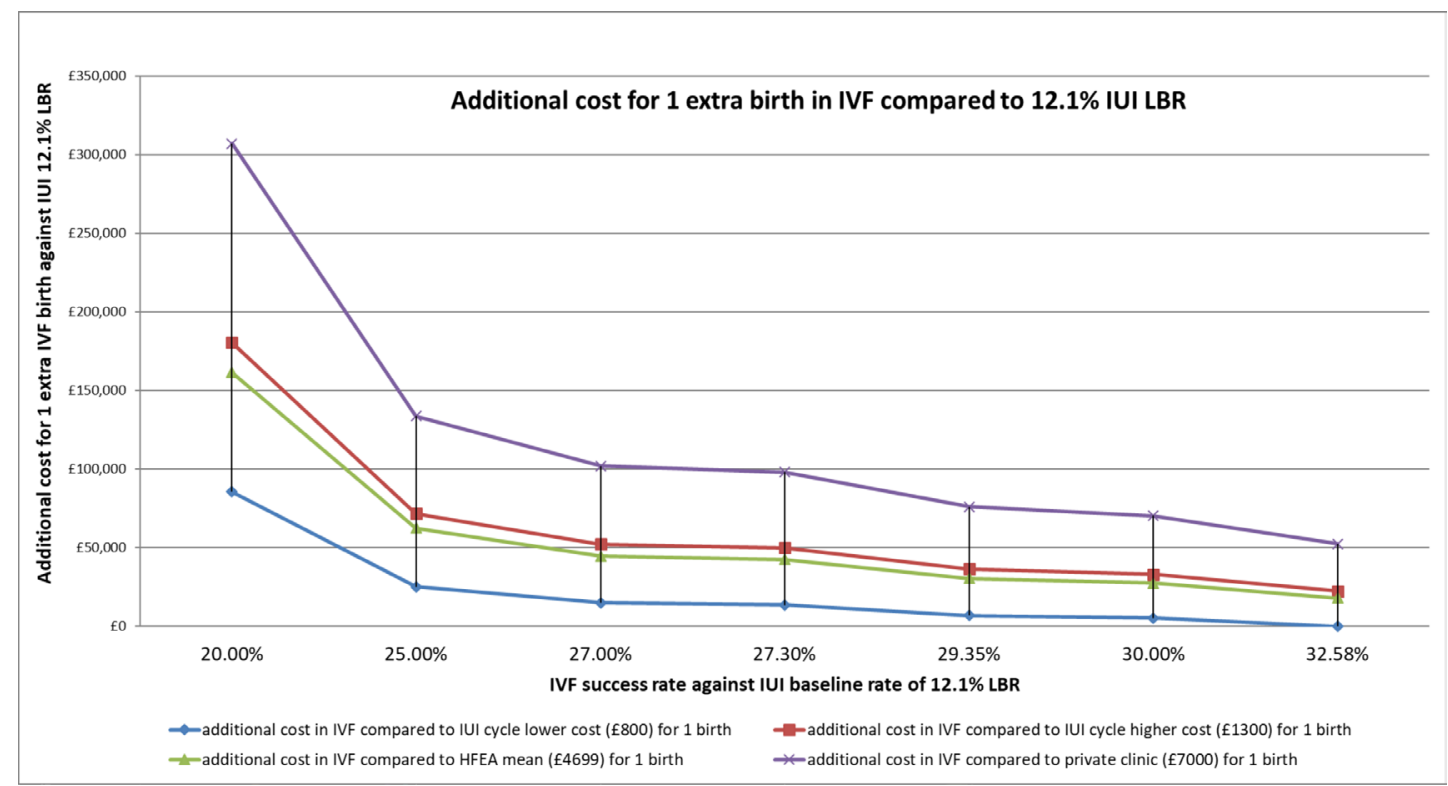

Figure 2 Economic and financial analyses of IUI versus IVF- direct cost-effective analyses to deliver 1 LB based on actual success rates and tariffs for IUI and IVF. Cost analyses with varying IVF success against mean IUI success. 
add-on techniques. ${ }^{19}$ IUI success rates appear to face downward pressures from IVF clinics by managing difficult cases through IUI. ${ }^{20}$

So far no reliable national figures exist for the levels of different risks for patients undergoing IVF and IUI to help them towards a journey of making informed decisions or to help clinicians counsel their patients. With this large database, the MGP rate as a proportion of all births was significantly higher following IVF than following IUI (RR: 1.45 (1.31 to 1.60 ), $\mathrm{p}<0.001$ ). This fact contradicts popular belief that IUI being the source of uncontrolled MGP. The rate of twins was also significantly higher following IVF (RR: 1.58, $\mathrm{p}<0.001$ ). There was $0.2 \%$ fetal reductions performed for IVF and none for IUI. Moderate to severe OHSS risk of $0.9 \%$ /birth or $0.25 \%$ per cycle was related to IVF only and not IUI (table 1). No maternal deaths were reported due to any complications but it can be argued the HFEA may not be fully informed as the death registry is not linked to the HFEA database. Terminations accounted for $0.81 \%$ of all total births (clinical pregnancy rates unavailable) in IVF and none in IUI. From 2012 to 2016 there were a total of 698 terminations evenly spread across this period. Medical reasons accounted for $73.6 \%$ of terminations with $22.4 \%$ of these being for Down's syndrome, and for the first time a $2.3 \%$ level of terminations for psychological and social reasons is revealed, while a further $24.1 \%$ of terminations were for undisclosed reasons. For the first time, direct information on level of terminations after IVF (but no terminations in IUI) conception is indicated for social and psychological reasons adding a new dimension of debate in ART. It serves to underline the vital role for support and counselling before, during and after fertility treatment.

The economics of the fertility industry provides important insights as to how financial considerations are overriding evidence-based medicine while suppressing the availability of low cost IUI. With regard to the financial information which can serve as important steers for patients and stakeholders in commissioning services emerge. The HFEA confirms the size of the IVF industry for 2016 to be worth around $£ 320000000$ in 2016, with a $61 \%$ growth in activity since $2007 / 2008$, revealing a mean tariff of $£ 4699 /$ cycle for IVF. ${ }^{13}$ The wider annual estimate of the fertility industry is $£ 500$ million which includes other activities such as cryopreservation of gametes and embryos, and add-on techniques. The main real tariffs paid by NHS commissioning groups for IUI were $£ 800$ and $£ 1300$ whereas for IVF $£ 3500-5000$ per cycle at the NHS level. The maternal and neonatal IVF risk burden of $£ 115082017$ representing $33 \%-50 \%$ of the 2016 IVF market value is passed on to the NHS. The level of NHS IVF funding from 2012 to 2016 was at $42.5 \%$, which is much higher than generally accepted, while a further $14.8 \%$ of IVF cycles were commissioned through private IVF practices. Private clinics are not public bodies and therefore are not accountable under the FOI Act. NHS funding for IUI in the UK was removed following the non-evidenced-based NICE guidelines ${ }^{2}$ although some commissioning groups continue funding IUI. This clearly needs to be reversed with immediate effect.

The unique algorithm (figures 1 and 2) reveals points of efficiencies and inefficiencies for IUI and IVF treatments and describes a spectrum of financial, economic and success relationships with each other. At baseline success rates for IUI (12.1\% LBR) and IVF (27.3\% LBR) (figure 1), ICER favoured IUI over IVF by at least $£ 13663$ to deliver $1 \mathrm{LB}$ against the cheapest IVF tariff. When the realistic mean IVF tariff of $£ 4699$ /cycle was considered mean IUI clinics could deliver a cost-effective benefit per LB of $£ 42558$ (figure 1). Better performing IUI clinic at $15.6 \%$ LBR clinic (NMUH) could extend the cost savings to $£ 76257$ per LB over mean IVF success of $27.3 \% \mathrm{LB}$ and tariff of $£ 4699$. The cost savings to achieve $1 \mathrm{LB}$ through improved IUI success over IVF are regarded as particularly high and beneficial to society. The algorithm defines the cost neutral point against mean IUI at $12.1 \%$ LBR, when IVF success reaches $32.58 \%$ (figure 2) which can be extended to $42 \%$ LBR for IVF with IUI at $15.6 \%$ LBR using the same algorithm. This threshold can also be much higher for higher IVF tariff. For every 1\% LB improvement of IUI, IVF success needs to improve by 2.7\% LBR to achieve the same incremental cost benefit as IUI. In other words, IVF needs to work much harder than IUI to achieve benefits and suggests the need to invest in improving IUI success rates. These ICER cost efficiency values should persuade stakeholders and patients to choose IUI before IVF. IVF success rates have improved by $2 \%$ only to $27.3 \%$ LBR from 2012 to 2016 despite expensive non-evidenced-based add-on techniques and treating potential IUI cases through IVF.

On the reverse scale, cost-effectiveness of IUI against lowest tariff IVF is maintained until the IUI success reaches $10.14 \%$ LBR (figure 1). Below $10.14 \%$ LB, IUI loses its cost-effectiveness against the lowest tariff IVF cycle. However, this lower IUI limit of $10.14 \%$ LBR will be even lower when considering higher tariff IVF (figure 1) and which can be extrapolated from figure 1 . Below these IUI low points practitioners need to re-evaluate their management, protocols or stop performing IUI.

Higher IVF success rate clinics are also associated with increased tariffs and MGP levels. A clinic with a 55\% LBR success has around $28 \% \mathrm{MGP},{ }^{10}$ and twice the normal UK MGP rate lose all benefits through increased tariffs ranging from $£ 7000$ to 15000 per cycle against mean IVF clinics. On tariff consideration alone, high-performing IUI clinic is more cost-effective than high-performing IVF clinics where tariff factors alone erode any benefits. Based on success and tariffs only, IUI at $15.6 \%$ (tariff $£ 800$ ) versus IVF at $27.3 \%$ ( $£ 4699$ ) versus IVF at $55 \%$ ( $£ 15$ $000)$ LBR would cost the patients $£ 5,128, £ 17404$ and $£ 27$ 273, respectively, to achieve $1 \mathrm{LB}$, notwithstanding the increasing risks for mother and babies along this sequence. The algorithm (figures 1 and 2) also advises that the most expensive high-performing IVF clinic at $55 \%$ LBR can match the mean IVF clinics at $27.3 \%$ LBR by dropping the tariff to $£ 9572 /$ cycle, provided these clinics 
can also reduce their MGP from $28 \%$ to $13.8 \%$ level. The financial analyses exclude the cost of OHSS, terminations and complication mainly for IVF, while there will be additional cost for drugs. Excluded also are the fees paid by IVF patients to cryopreserve embryos, back-up sperm or purchase add-on procedures.

Separate ICER calculations for high-performing IUI (15.6\% LBR, £800 tariff) against high-performing IVF ( $55 \%$ LBR, $£ 15000$ ) show that it is $£ 56204$ cheaper to gain 1 LB by IUI. Likewise, separate ICER calculations for mid-performing IVF (27\% LBR, £4699) against high-performing IVF (55\% LBR, £15 000) show that it is $£ 35246$ cheaper to gain $1 \mathrm{LB}$ through mid-IVF clinics compared with high-performing IVF clinics.

The algorithms allow detailed cross analyses of the practices and confirm IUI is superior to IVF to derive the best possible cost benefit to gain a child while minimising the risk to mother and babies if applicable. The second line option is for patients to choose a mid-performing IVF clinic paying attention to MGP levels. Evidencedbased bodies have a duty of care to explicitly inform stakeholders when ICER is favourable, and in this case IUI is dominant over IVF. ${ }^{21}$ Previous cost-effective studies have been performed against extremely poor IUI results, while relying on optimal IVF in local settings to persuade purchasers to fund more IVF cycles instead of IUI. ${ }^{22} 23$

The second major cost analyses relate to the MGP maternal/neonatal-related cost burden which has remained invisible to date. For 2016, the IVF cost burden to the NHS was $£ 115$ million ( $£ 532$ million over 20122016), against the IVF clinics turnover estimated between $£ 340$ and 538 million depending on the tariffs range $£ 3500-5000 /$ per cycle cost considered. The HFEA mean cost estimate per cycle treatment for 2016 was £4699/ cycle and a market worth of $£ 320$ million. In contrast the IUI market worth was £3.24-5.3 million using tariffs of $£ 800-1300$, against the negative cost impact of $£ 2.94$ million for 2016. It is prudent to fund only essential IVF such as bilateral tubal blockages and severe male factor infertility as a priority before considering IUI failed cases through modified eSET (elective single embryo transfer) in order to control costs while minimising MGP. ${ }^{24}$

There are some critical limitations to the current study and the main one being it was an observational and retrospective in nature, with the main concern being selection bias of data collection. However, due to the unique nature of having gained the treatment cycles data under FOI from the UK regulatory body, the potential for selection bias is minimised. The data do not reveal the baseline characteristics and treatment details of patients such as age, cause, type and duration of subfertility, prognostic indicators like ovarian reserve status, stimulation protocols, fresh or frozen IVF cycles, the distinction between IVF or ICSI or the number of cycles of treatment each patient has undergone to be able to comment on the cumulative success rates. Further limitation of this study relates to non-random selection of IUI or IVF treatment in this study with good prognosis patients may have been selected for IUI or IVF as first choice. Contrastingly, some poor prognosis patients may have chosen lesser invasive IUI treatment as the only option and some have had IVF cycle cancellation with subsequent rescue IUI cycles, both of which may potentially bias IUI and IVF success rates. Specific patient pathologies such as severe male factor infertility or bilateral tubal blockages will obviously necessitate IVF procedures creating a degree of selection bias but this cannot be revealed from our data analyses. However, it is important to recognise that by analysing aggregate national UK data for the entire subfertile population rather than a sample of the population, the risks of selection and treatment biases are somewhat mitigated and potentially allowing a high degree of data generalisability. Stakeholders in other countries should review their practices, risks and costs of assisted reproductive procedures based on our UK experience.

Patients and stakeholders also need to consider intervention-related risks and long-term risks before making choices. IUI and IVF both have common underlying risks relating to the general health pathologies of subfertile couples and due to ovarian stimulation protocols. However, there appear long-term added risks for babies from IVF, ICSI, embryo culture and freezing procedures. The added risks have been reported for singleton IVF babies and singletons after fetal reduction having pre-term weights, and large sized babies from frozen embryo procedures. Late onset diseases relating to increased risk of some cancers are being reported. ${ }^{25} 26$ In vitro embryo cultures and exposures may affect later life developments along with any (epi) genetic modifications provide added risks ${ }^{27}$ or lead to higher imprinting disorders such as Beckwith-Wiedemann syndrome ${ }^{28-30} 30$ compared with naturally conceived children. This is a dynamic and evolving area of research.

In conclusion, IUI LBRs are much closer to IVF than previously described. IUI is associated with lesser risks to mothers and babies and more cost-effective than IVF in delivering $1 \mathrm{LB}$ against current tariffs. IUI always has lower overall costs than IVF in commissioning treatment to achieve $1 \mathrm{LB}$. The cost burden to the healthcare system is excessive for IVF. Some selection bias cannot be excluded to the retrospective design and the data should be interpreted with caution. However, this unfettered, uniquely integrated analysis of success, risks and cost provides important information to healthcare stakeholders and governments to develop effective fertility treatment policies.

\section{Author affiliations}

${ }^{1}$ Reproductive Medicine Clinic, North Middlesex University Hospital NHS Trust, London, N18 1QX, UK

${ }^{2}$ Homerton Fertility Unit, Homerton University Hospital, Homerton Row, London E9 6SR, UK

${ }^{3}$ Department of Health Sciences, Faculty of Science, Vrije Universiteit Amsterdam, Amsterdam Public Health research institute, Amsterdam, The Netherlands ${ }^{4}$ Research Institute Reproduction and development, University Medical Centers Amsterdam, location VUmc and AMC, De Boelelaan 1081, 1081 HV Amsterdam, The Netherlands 
${ }^{5}$ University Hospitals of Derby and Burton NHS Trust, Royal Derby Hospital, Derby, Derby, UK

${ }^{6}$ Linacre College, Oxford University, St. Cross Road, Oxford, 0X13JA, UK ${ }^{7}$ EGA Institute for Womens Health, Faculty of Population Health Science, University College London, London, WC1E 6HX, UK

Contributors GB and RH framed the hypothesis, $\mathrm{PR}$ undertook the freedom of information requests, $\mathrm{PH}$ and $\mathrm{KJ}$ performed statistical analyses independently of each other, RA developed the financial models and algorithms. GB, RH (Professor), JAFH (Professor and Head of gynaecological department), PH, KJ, IK, Al (Specialist IUI nurse), JB (Professor of Healthcare Economics), AA-H, EJ provided clinical information and critical analyses throughout. All authors helped prepare and reviewed the final manuscript.

Funding The authors have not declared a specific grant for this research from any funding agency in the public, commercial or not-for-profit sectors.

Competing interests None declared.

Patient consent for publication Not required.

Provenance and peer review Not commissioned; externally peer reviewed.

Data availability statement All data relevant to the study are included in the article or uploaded as supplementary information. Source data are available from the UK Human Fertilisation and Embryology Authority regulatory body. https://www. hfea.gov.uk/choose-a-clinic/clinic-search/; https://www.hfea.gov.uk/about-us/ publications/research-and-data/. Freedom of information gained is as presented within the manuscript.

Open access This is an open access article distributed in accordance with the Creative Commons Attribution Non Commercial (CC BY-NC 4.0) license, which permits others to distribute, remix, adapt, build upon this work non-commercially, and license their derivative works on different terms, provided the original work is properly cited, appropriate credit is given, any changes made indicated, and the use is non-commercial. See: http://creativecommons.org/licenses/by-nc/4.0/.

ORCID iDs

Gulam Bahadur http://orcid.org/0000-0002-0136-9278

Eric Jauniaux http://orcid.org/0000-0003-0925-7737

\section{REFERENCES}

1 HFEA Jan. What's the difference between IVF and IUI? 2019. Available: https://www.hfea.gov.uk/treatments/explore-alltreatments/intrauterine-insemination-iui/

2 NICE. National Institute for Health and Care Excellence. Fertility problems: assessment and treatment. 2013. Clinical guideline [CG156] Published date: February 2013 Last updated: August 2016, 2013. Available: https://www.nice.org.uk/guidance/cg156

3 Bahadur G, Woodward B, Homburg R, et al. Pitfalls of NICE recommendations on fertility treatment. BMJ 2017;356:j751.

4 Cissen M, Bensdorp A, Cohlen BJ, et al. Assisted reproductive technologies for male subfertility. Cochrane Database Syst Rev 2016;2:CD000360.

5 Farquhar CM, Liu E, Armstrong S, et al. Intrauterine insemination with ovarian stimulation versus expectant management for unexplained infertility (TUI): a pragmatic, open-label, randomised, controlled, twocentre trial. Lancet 2018;391:441-50.

6 Nandi A, Bhide P, Hooper R, et al. Intrauterine insemination with gonadotropin stimulation or in vitro fertilization for the treatment of unexplained subfertility: a randomized controlled trial. Fertil Steril 2017;107:1329-35.

7 Bensdorp AJ, Tjon-Kon-Fat RI, Bossuyt PMM, et al. Prevention of multiple pregnancies in couples with unexplained or mild male subfertility: randomised controlled trial of in vitro fertilisation with single embryo transfer or in vitro fertilisation in modified natural cycle compared with intrauterine insemination with controlled ovarian hyperstimulation. BMJ 2015;350:g7771.

8 Tjon-Kon-Fat RI, Tajik P, Zafarmand MH, et al. Ivf or IUI as first-line treatment in unexplained subfertility: the conundrum of treatment selection markers. Hum Reprod 2017;32:1028-32.
9 Ferraretti AP, Nygren K, Nyboe Andersen A, et al. The European IVF monitoring Consortium (EIM), for the European Society of human reproduction and embryology (ESHRE). trends over 15 years in art in Europe: an analysis of 6 million cycles. Hum Reprod Open 2017;2:1-10

10 data H. Choose a clinic, 2019. Available: https://www.hfea.gov.uk/ choose-a-clinic/

11 Ledger WL, Anumba D, Marlow N, et al. Cost of multiple births Study Group (combs group). The costs to the NHS of multiple births after IVF treatment in the UK. BJOG 2006;113:21-5.

12 Bouwsma EVA, Bosmans JE, van Dongen JM, et al. CostEffectiveness of an Internet-based perioperative care programme to enhance postoperative recovery in gynaecological patients: economic evaluation alongside a stepped-wedge cluster-randomised trial. BMJ Open 2018;8:e017782.

13 Feb H. Market value of IVF industry, 2019. Available: https://www. hfea.gov.uk/media/2697/2018-11-14-authority-papers-final-website. pdf

14 Bahadur G, Almossawi O, Zeirideen Zaid R, et al. Semen characteristics in consecutive ejaculates with short abstinence in subfertile males. Reprod Biomed Online 2016;32:323-8.

15 Danhof NA, van Wely M, Repping S, et al. Follicle stimulating hormone versus clomiphene citrate in intrauterine insemination for unexplained subfertility: a randomized controlled trial. Hum Reprod 2018;33:1866-74.

16 Goverde AJ, McDonnell J, Vermeiden JP, et al. Intrauterine insemination or in-vitro fertilisation in idiopathic subfertility and male subfertility: a randomised trial and cost-effectiveness analysis. Lancet 2000;355:13-18.

17 Tjon-Kon-Fat RI, Bensdorp AJ. And Bossuyt PMM: is IVF-served two different ways-more cost-effective than IUI with controlled ovarian hyperstimulation? Hum Reprod 2015;10:2331-9.

18 Geisler ME, Ledwidge M, Bermingham M, et al. Intrauterine insemination-No more Mr. N.I.C.E. Guy? Eur J Obstet Gynecol Reprod Biol 2017;210:342-7.

19 Heneghan C, Spencer EA, Bobrovitz N, et al. Lack of evidence for interventions offered in UK fertility centres. BMJ 2016;355:i6295.

20 Delbos L, Parot-Schinkel E, El Hachem H, et al. ConFIRM trial - conversion of in vitro fertilization cycles to intrauterine inseminations in patients with a poor ovarian response to stimulation: a protocol for a multicentric, prospective randomized trial. Trials 2018;19:565.

21 NICE. Developing NICE guidelines: the manual process and methods published: 31 October 2014, 2014. Available: nice.org.uk/process/ pmg20

22 Pashayan N, Lyratzopoulos G, Mathur R. Cost-Effectiveness of primary offer of IVF vs. primary offer of IUI followed by IVF (for IUI failures) in couples with unexplained or mild male factor subfertility. BMC Health Serv Res 2006;6:80.

23 Wordsworth S, Buchanan J, Mollison J, et al. Clomifene citrate and intrauterine insemination as first-line treatments for unexplained infertility: are they cost-effective? Hum Reprod 2011;26:369-75.

24 Groen H, Tonch N, Simons AHM, et al. Modified natural cycle versus controlled ovarian hyperstimulation IVF: a cost-effectiveness evaluation of three simulated treatment scenarios. Hum Reprod 2013;28:3236-46.

25 Spector LG, Brown MB, Wantman E, et al. Association of in vitro fertilization with childhood cancer in the United States. JAMA Pediatr 2019;173:e190392.

26 Spaan M, van den Belt-Dusebout AW, van den Heuvel-Eibrink MM, et al. Risk of cancer in children and young adults conceived by assisted reproductive technology. Hum Reprod 2019;34:740-50.

27 Roseboom TJ. Developmental plasticity and its relevance to assisted human reproduction. Hum Reprod 2018;33:546-52.

28 DeBaun MR, Niemitz EL, Feinberg AP. Association of in vitro fertilization with Beckwith-Wiedemann syndrome and epigenetic alterations of LIT1 and H19. Am J Hum Genet 2003;72:156-60.

29 Gicquel C, Gaston V, Mandelbaum J, et al. In vitro fertilization may increase the risk of Beckwith-Wiedemann syndrome related to the abnormal imprinting of the KCN1OT gene. Am J Hum Genet 2003:72:1338-41.

30 Hiura $\mathrm{H}$, Okae H, Miyauchi $\mathrm{N}$, et al. Characterization of DNA methylation errors in patients with imprinting disorders conceived by assisted reproduction technologies. Hum Reprod 2012;27:2541-8. 